\title{
Espaço de promoção da saúde na graduação em Odontologia: (re)significando saberes e práticas na produção do cuidado
}

\author{
Janaína Rocha de Sousa Almeida*; Maria Cláudia de Freitas Lima**; Camila Castro***; Carlos \\ Eduardo de Sousa Praxedes**; Maria Elisabeth Sousa Amaral****; Renata Mota Rodrigues Bitu \\ Sousa $* * * * *$
}

* Doutora em Clínica Odontológica, docente, curso de graduação em Odontologia do Centro Universitário Christus

** Mestre em Saúde da Família, docente, curso de graduação em Odontologia do Centro Universitário Christus

*** Doutora em Saúde Coletiva, docente, curso de graduação em Odontologia do Centro Universitário Christus

**** Mestre em Health Management, docente, curso de graduação em Odontologia do Centro Universitário Christus

***** Doutora em Saúde Coletiva, Coordenadora do curso de graduação em Odontologia do Centro Universitário Christus

Recebido em 16/07/2018. Aprovado em 29/06/2019.

\begin{abstract}
RESUMO
A promoção da saúde demanda que a prática da atenção à saúde transcenda os conceitos normativos da Medicina em suas expressões clínicas e/ou de saúde pública, precisando ser construída e reconstruída em reconhecimento da realidade das experiências de vida dos pacientes. Desse modo, o objetivo desse artigo é relatar a experiência de desenvolvimento de atividades de promoção de saúde e prevenção de doenças em um espaço compartilhado da Clínica Escola de Odontologia do Centro Universitário Christus, no período de 2015 a 2017. Os grupos de trabalhos foram formados por docentes, discentes e pacientes que eram atendidos na clínica. As ações realizadas foram pactuadas com os usuários no primeiro acesso à clínica, ao iniciar o atendimento do paciente, no âmbito da proposta de construção partilhada do plano de cuidado. Dentre as temáticas trabalhadas destacam-se diabetes e hipertensão, nutrição, saúde da mulher, saúde mental, disfunção temporomandibular, medicalização da vida e automedicação, sexualidade e infecções sexualmente transmissíveis, lesões orais, dores posturais, cuidados e prevenção de acidente vascular encefálico, meio ambiente, sustentabilidade, cárie e doença periodontal. O Espaço de Promoção da Saúde como estratégia metodológica desenvolvida na graduação em Odontologia tem propiciado uma formação acadêmica diferenciada, na qual os discentes e docentes sistematizam o planejamento, trabalham em equipe e promovem ações criativas, problematizadoras, participativas e interprofissionais, experienciando a vivência intersubjetiva com coletivos.
\end{abstract}

Descritores: Saúde Bucal. Promoção da Saúde. Educação. 


\section{INTRODUÇÃO}

A saúde tem determinações sociais, econômicas, políticas e culturais mais amplas do que simplesmente a herança genética, a biologia humana e os fatores ambientais mais imediatos. De fato, bases sólidas de evidências foram demonstrando que existe uma forte relação entre os estilos de vida das pessoas, sua posição social e econômica, suas condições de vida e seu estado de saúde. Essa compreensão, por parte dos diversos atores, também levou ao desenvolvimento de metodologias e práticas no contexto da saúde de modos e formas que podem vir a modificar esses determinantes estruturais e pessoais da saúde ${ }^{1,2}$.

Nessa perspectiva, a promoção da saúde demanda que a prática da atenção à saúde transcenda os conceitos normativos da Medicina em suas expressões clínicas e/ou de saúde pública, precisando ser construída e reconstruída em reconhecimento da realidade das experiências de vida dos pacientes, do significado existencial das suas condições de vida e situações ${ }^{3,4}$.

Assim, as ações de promoção da saúde condizentes com a realidade em que vivemos, devem ser orientadas para a busca de uma melhor qualidade da vida individual e coletiva, porém uma qualidade não centrada apenas em referenciais técnicos ou econômicos, mas sim uma qualidade orientada para o desenvolvimento integral do ser humano ${ }^{4,5}$.

Nesse sentido, a estratégia de promover saúde vem sendo reorientada para a superação de práticas restritas ao modelo de prevenção, ressaltando-se que a boca se constitui como parte do corpo, fazendo a mediação dos sujeitos com o mundo. Portanto, faz-se necessário desenvolver estratégias que incorporem a relação sujeito-corpodesejo, fomentando o autocuidado como experiência singular de sentir-se saudável ou doente $^{6}$.

Analisando o universo da Odontologia, este desenho requer uma reconstrução teórico- metodológica da clínica, ainda centrada nas demandas cirúrgicas identificadas pelo profissional. Nessa perspectiva surge o Espaço de Promoção da Saúde (EPS) como parte da Clínica Odontológica Ampliada que integra o curso de graduação em Odontologia do Centro Universitário Christus.

O EPS tem como objetivos desenvolver com os discentes outras habilidades, competências e atitudes no processo de formação em Odontologia, como por exemplo trabalhar educação em saúde com coletivos, ancorada num planejamento de ações que propiciem a interação e participação dos sujeitos implicados, fomentar a realização de ações interdisciplinares e interprofissionais, exercitar a promoção da saúde como parte integrante do plano de cuidado, visando à integralidade do cuidado e o desenvolvimento da autonomia e do protagonismo dos participantes.

Desse modo, os princípios de organização do ESP se constituem em participação, autonomia, integralidade, protagonismo e horizontalidade nas relações. Diante da necessidade de se fortalecer as ações de promoção à saúde e prevenção de doenças, no contexto da formação em saúde, a proposta desse artigo é relatar a experiência do EPS na graduação em Odontologia.

\section{RELATO DA EXPERIÊNCIA}

Trata-se de um relato de experiência resultante das atividades desenvolvidas no Espaço de Promoção de Saúde relativas ao período de 2015 a 2017, que tem como cenário o espaço da Clínica Escola de Odontologia do Centro Universitário Christus.

Os grupos de trabalhos foram formados por docentes, discentes e pacientes que eram atendidos na Clínica Escola de Odontologia. Foram incluídos no EPS estudantes da graduação em Odontologia do primeiro ao oitavo semestres, cursando as disciplinas de Bioquímica, Saúde-Educação e Sociedade, Clínicas I, II e III visando à atenção à 
saúde de adultos.

Quanto aos docentes, participaram os responsáveis pelas disciplinas clínicas e de Saúde Coletiva, bem como, docentes de outros cursos da instituição, tais como Fisioterapia, Psicologia, Enfermagem e Nutrição, somando-se 24 profissionais. Do quantitativo de pacientes que estavam em atendimento odontológico no decorrer dos anos 2015 a 2017, 1.338 adultos participaram das atividades no EPS (quadro 1).

As ações realizadas são pactuadas com os usuários no primeiro acesso à clínica, ao iniciar o atendimento, no âmbito da proposta de construção partilhada do plano de cuidado. Os estudantes são orientados a explicar e convidar seus pacientes a participar do EPS. Em sua primeira participação realiza-se um levantamento das expectativas dos pacientes, considerando os temas em saúde relevantes para cada grupo. Segue-se a isso o planejamento das ações de educação em saúde que irão acontecer, nas quais discentes e docentes trabalham em equipe construindo uma atividade lúdica, participativa e que estabeleça subsídios para processos de transformação.

As atividades ocorrem quinzenalmente e a pactuação é que o paciente participe das ações, no mínimo três vezes durante a execução do seu plano de cuidado odontológico. O paciente é agendado para participar de atividade no EPS e nesse dia ele não será submetido ao atendimento clínico, mas cumprirá parte do seu plano de cuidado desenvolvendo atividades de promoção de saúde.

Nas atividades desenvolvidas no EPS considera-se o entendimento que para promover a saúde por meio de ações de educação em saúde precisamos considerar os modos de viver, de pensar e se relacionar com a realidade, bem como os determinantes sociais em saúde que compõem os contextos dos participantes. Nessa direção, se faz fundamental compreender o paciente como sujeito do processo e o contexto em que vive como aspectos norteadores do processo do cuidar, ampliando a escuta e o fazer clínico, centrado no diálogo. Isso favorece o compartilhar de saberes e práticas, estabelecendo elementos que fomentam o (re)pensar em prol de um cuidado integral.

Diversas temáticas foram desenvolvidas no EPS como: diabetes e hipertensão, nutrição, saúde da mulher, saúde mental, disfunção temporomandibular, medicalização da vida e automedicação, sexualidade e infecções sexualmente transmissíveis, lesões orais, dores posturais, cuidados e prevenção de acidente vascular encefálico (AVE), meio ambiente, sustentabilidade, cárie e doença periodontal.

Destaca-se o fomento à realização de ações interprofissionais integradas com outros cursos da instituição, como Enfermagem, Fisioterapia, Psicologia, Nutrição e a pactuação das atividades considerando também as campanhas nacionais desenvolvidas pelo Ministério da Saúde.

A diabetes e hipertensão arterial foram trabalhadas por meio de atividades realizadas juntamente com os acadêmicos de Nutrição, que abordavam a alimentação saudável e sua importância para a saúde do indivíduo. Nesta atividade os pacientes, após uma dinâmica de apresentação na qual sinalizavam a sua prática cotidiana, passaram por uma simulação de compras em supermercados e preparação de alimentos. Nesse cenário foram construídas reflexões em torno da alimentação saudável e sua relação com a diabetes e hipertensão, em consonância com o contexto de cada participante. A atividade também contemplou aferição de pressão e glicemia. Essa ação também foi desenvolvida de forma dialógica, na qual os pacientes socializavam seu conhecimento prévio, bem como suas dúvidas. Vale ressaltar que, de acordo com os resultados observados, os pacientes receberam as orientações necessárias.

Referente às ações sobre a saúde da mulher, os discentes e docentes preparam um momento envolvendo a prevenção de câncer do colo do útero 
e de mama, tratamento hormonal, menopausa, tensão pré-menstrual, violência doméstica e direitos das mulheres, autoestima e autocuidado e suas relações com a saúde bucal. A atividade foi realizada de forma interativa, por meio de um jogo com perguntas e respostas.

A saúde mental foi trabalhada em roda de conversa, abordando a prevenção sobre o suicídio, depressão, estresse e ansiedade, buscando conexões com a saúde bucal, relacionando tensão, ansiedade e a disfunção temporomandibular e dor orofacial. Nesta atividade os pacientes passaram por sessões de relaxamento e meditação como práticas integradas que visam contribuir para melhoria da saúde mental.

As temáticas, medicalização da vida e automedicação foram trabalhadas por meio de uma peça de teatro encenada pelos discentes, a qual retratou a situação de uma adolescente que ao chegar a casa chorando muito porque tinha acabado o namoro, recebeu da mãe uma dose elevada de medicamento, o que resultou em diversos efeitos colaterais, como risos exagerados, tonturas, letargia, seguida de sono por mais 15 horas. Ao acordar, a adolescente relatou não lembrar o episódio após a medicação, mas apresentava forte dor de cabeça. Frente a esse cenário, realizou-se uma roda de conversa com os participantes visando problematizar a temática e identificar ações que propiciassem possibilidades saudáveis de se lidar com a questão da automedicação e da medicalização da vida.

A sexualidade foi abordada após uma dinâmica de apresentação e integração relativa ao tema, destacando a concepção de sexualidade e a relação com os usos da boca. A ação também evidenciou, por meio de uma exposição dialogada, as manifestações orais das infecções sexualmente transmissíveis e os meios de prevenção.

Outra temática que gerou expressiva interação entre os participantes consistiu numa atividade desenvolvida em parceria com docentes e discentes do curso de Fisioterapia, na qual foram abordadas as dores geradas pela postura inadequada no ambiente de trabalho, a importância da massoterapia como prática de cuidado a saúde e prevenção do AVE. Essa atividade também disponibilizou para os pacientes a realização de massagens terapêuticas, promovidas pelos técnicos do curso de Massoterapia.

Com o objetivo de promover uma reflexão sobre o meio ambiente e a sustentabilidade, os temas lixo e cidadania foram abordados de forma interativa, contextualizados numa roda de conversa. Após o diálogo realizou-se uma oficina de reutilização de garrafas plásticas, evidenciando um modo criativo e prático para reaproveitamento de materiais recicláveis.

As doenças cárie e periodontal também foram trabalhadas utilizando questões problematizadoras, textos e recortes de jornais consolidados em livretos construídos pelos discentes, e apresentados aos participantes para realização de trabalho em grupos. Cada grupo recebeu inicialmente uma questão problematizadora e após o diálogo, apresentou uma síntese com relação às temáticas cárie e doença periodontal, como também relatou as dúvidas que permearam a conversa. Os livretos foram utilizados como complementos da atividade. A ação também contemplou a realização de um momento de autocuidado, considerando que o EPS dispõe de um local apropriado para aperfeiçoamento da higiene oral, no qual os pacientes se autoavaliaram com relação à saúde bucal por meio da evidenciação de biofilme, escovação e uso de fio dental.

No tocante às atividades desenvolvidas de forma interdisciplinar, faz-se oportuno destacar as que são realizadas com os estudantes do $1^{\circ}$ semestre de Odontologia, que juntamente com os professores de Bioquímica planejam e realizam ações relevantes no EPS referentes à composição dos alimentos e a relação com a saúde. Parte dessas 
atividades são executadas tendo como base a organização de uma mesa composta com alguns dos alimentos mais comuns que fazem parte do cotidiano alimentar do cearense, como por exemplo, o cuscuz, café, batata doce e tapioca. Nesse contexto, os discentes promovem um diálogo considerando as vivências e as práticas alimentares/nutricionais dos participantes. Esses encontros propiciam o primeiro contato dos discentes com os pacientes e com as ações de educação em saúde, experienciando o exercício da escuta e do trabalho coletivo, gerando novas concepções para a formação em Odontologia.

Nesse período de vivências pedagógicas no EPS, tem-se buscado desenvolver estratégias metodológicas que favoreçam a integração, a criatividade, a partilha e a construção do conhecimento de forma lúdica e interativa. Ressalta-se, nessa compreensão, que o EPS também tem contribuído para despertar as potencialidades dos discentes, oxigenando a autonomia e o protagonismo.

Quadro 1. caracterização dos participantes do EPS no período de 2015 a 2017

\begin{tabular}{|c|c|c|c|}
\hline $\begin{array}{c}\text { Faixa etária } \\
\text { dos pacientes }\end{array}$ & $\begin{array}{c}\text { Número de } \\
\text { Pacientes }\end{array}$ & $\begin{array}{c}\text { Número de } \\
\text { Discentes }\end{array}$ & $\begin{array}{c}\text { Número de } \\
\text { Docentes }\end{array}$ \\
\hline $15-70$ anos & 1.338 & 347 & 24 \\
\hline
\end{tabular}

O EPS é, sobretudo, uma aposta na construção pedagógica da Clínica Ampliada, sendo essa uma das diretrizes da Política Nacional de Humanização e se propõe a qualificar o modo de se produzir saúde, ampliando a clínica e desenvolvendo a autonomia do usuário do serviço de saúde, da família e da comunidade ${ }^{7}$.

Ao envolver, no mesmo espaço, pacientes, docentes e discentes numa atividade fora dos padrões de um atendimento odontológico convencional, amplia-se o olhar técnico, abre-se espaço para a escuta e busca de outros modos de trabalhar com as demandas dos usuários.

Trabalhar a promoção de saúde e a prevenção de doenças dentro desta perspectiva implica não ser refém de protocolos rígidos, compreendendo as singularidades dos sujeitos, acolhendo as subjetividades e abrindo espaço para as possíveis inovações. Esta visão choca-se com a clínica tradicional na qual existe uma centralidade nos aspectos biológicos em detrimento das questões sociais de cada indivíduo envolvido ${ }^{8}$.
Com base nesta compreensão, busca-se vivenciar por meio do EPS uma prática de promoção da saúde autêntica, horizontal, engajada e aberta para captar as necessidades dos usuários. Tratando-se de uma vivência interdisciplinar capaz de provocar mudanças na docência e na prática cotidiana do curso de odontologia, contribui para uma experiência acadêmica amplificada e que possibilita a formação de um profissional apto a entender que sua ação pode e deve ser abrangente e transformadora.

Nesse contexto, urge a necessidade de ruptura com processos de formação em Odontologia que conservam o afastamento entre técnicas odontológicas e o cuidado integral. Faz-se essencial promover a formação de profissionais com competências e habilidades para uma prática humanizada e de produção integral do cuidado, visando responder as necessidades da população, tanto na perspectiva individual quanto coletiva ${ }^{8}$.

A necessidade dessa formação diferenciada tornou-se mais evidente com as mudanças das 
diretrizes curriculares dos cursos da área da saúde, sendo necessária a utilização de estratégias educacionais inovadoras que contribuam para a formação de um discente capaz de manejar as tecnologias duras e leves com excelência. Este esforço traduz-se na construção de capacidades para manejar elementos materiais e não materiais no exercício da produção do cuidado ${ }^{9}$.

Ressalta-se que o trabalho de forma interdisciplinar em equipes multiprofissionais caracteriza-se como um desafio na graduação em odontologia. A construção dessa habilidade envolve a construção de competências relacionadas à comunicação, liderança e capacidade de tomar decisões, sendo capaz de escutar, acolher e construir vínculos com seus usuários ${ }^{10}$. O EPS torna-se assim, importante ferramenta para a formação de um cirurgião-dentista capaz de cuidar de seu paciente de forma integrada.

Do ponto de vista da metodologia utilizada para trabalhar as atividades de promoção de saúde e prevenção de doenças é interessante pontuar que cada tema trabalhado no EPS se torna objeto de reflexão e estudo para o grupo de discentes que conduzirá a atividade com os usuários e docentes. Esta atividade por si só contribui para a construção de novas perspectivas de atuação do cirurgiãodentista e consequentemente para a construção de uma Clínica Ampliada que enfrenta o desafio de encarar com seriedade os sujeitos: o cuidador/profissional e o que é cuidado ${ }^{11}$.

Destaca-se que o lúdico é ferramenta utilizada nas diversas atividades, buscando dessa forma trabalhar o sentimento, a emoção e imaginação dos participantes. O lúdico favorece relações reflexivas, inteligentes e socializadoras, assim o ato de educar torna-se mais interessante e significativo, trazendo prazer e satisfação pessoal $^{12}$.

A metodologia utilizada parte do princípio construtivista implementado por Paulo Freire ${ }^{13,14}$ na qual as atividades partem dos saberes prévios dos participantes, seguindo-se a construção coletiva do conhecimento - o EPS vai tecendo novos modos de produção do cuidado. O planejamento dos encontros é realizado baseandose em uma metodologia participativa e problematizadora, levando-se em consideração o perfil dos participantes, bem como o protagonismo dos discentes na elaboração das atividades que serão realizadas.

Trabalhar temáticas que tenham uma relação com a saúde de forma ampliada é certamente um grande diferencial das atividades realizadas no EPS. Tal fato, possibilita que não apenas as questões biológicas dos indivíduos sejam trabalhadas, respeitando outros aspectos que estejam relacionados com o sujeito, utilizando uma postura ética que implica na escuta do usuário em suas queixas ${ }^{8}$.

Frente a essas considerações, evidencia-se que $\mathrm{o}$ percurso pedagógico do EPS tem proporcionado aspectos essenciais e inovadores ao processo de graduação em Odontologia, e dentre eles, constata-se a efetivação de ações de atenção à saúde centrada na pessoa, respeitando suas potencias e vulnerabilidades.

Vale destacar que o contato dos discentes com realidades complexas relatadas pelos pacientes no EPS favorece a compreensão de que se faz imprescindível a realização de práticas de cuidado, que considere a realidade socioeconômica e cultural, propiciando a construção de um plano de cuidado numa perspectiva de atenção integral à saúde.

\section{CONSIDERAÇÕES FINAIS}

O EPS como estratégia metodológica desenvolvida na graduação em Odontologia tem propiciado uma formação acadêmica diferenciada, na qual os discentes e docentes sistematizam o planejamento, trabalham em equipe e promovem ações criativas, problematizadoras, participativas e interprofissionais experienciando a vivência 
intersubjetiva com coletivos.

Apreende-se que a incorporação do sujeito na construção de espaços de promoção da saúde tem efetividade diferenciada pelo envolvimento do individuo com o seu autocuidado e a sua reabilitação. Nesse sentido, o EPS se materializa como estratégia pedagógica, favorecendo a concepção ampliada de saúde numa perspectiva de (re)significação das práticas de produção do cuidado, considerando nesse contexto o fortalecimento da autonomia e o protagonismo dos discentes.

O desenvolvimento das ações no EPS evidencia um impacto relevante na mudança histórica do paradigma tecnicista na graduação em Odontologia, reverberando potencias para o fortalecimento da promoção da saúde e contribuindo para uma formação ancorada numa concepção ampliada de saúde, de forma ética, crítica e proativa, propiciando assim novos sentidos na produção integral do cuidado.

\section{ABSTRACT}

Health promotion space in undergraduate Dentistry courses: (re)signifying knowledge and practices in the care production

Health promotion requires that the health care practice transcends the normative concepts of Medicine in its clinical and/or public health expressions, and needs to be constructed and reconstructed in recognition of the reality of patients' life experiences. Thus, the objective of this article is to report the experience of developing activities of health promotion and disease prevention in a shared space of the School of Clinical Dentistry of Christus University Center, from 2015 to 2017. The work groups were formed by teachers, students and patients who were assisted at the clinic. The actions carried out were agreed with the users in the first access to the clinic, when starting the patient care, within the framework of the proposal of shared construction of the care plan. Among the individuals studied are diabetes and hypertension, nutrition, women's health, mental health, temporomandibular disorder, medicalization of life and self-medication, sexuality and sexually transmitted infections, oral lesions, postural pain, care and prevention of stroke, environment, sustainability, caries and periodontal disease. The Health Promotion Space as a methodological strategy developed in the undergraduate degree in Dentistry has provided a differentiated academic training in which students and teachers systematize planning, work as a team and promote creative, problematizing, participatory and interprofessional actions, experiencing intersubjective experience with collectives.

Descriptors: Oral Health. Health Promotion. Education.

\section{REFERÊNCIAS}

1. Czeresnia D, De Freitas CM. Promoção da saúde: conceitos, reflexões, tendências. Editora FIOCRUZ, 2009.

2. Silva JV, Oliveira AGRC. Individual and contextual factors associated to the selfperception of oral health in Brazilian adults. Rev Saúde Pública. 2018; 52:29. [Acesso em: 10 de março de 2018]. Disponível em: http://www.scielo.br/scielo.php?script=sci_ar ttext\&pid=S0034$\underline{89102018000100223 \& \operatorname{lng}=\mathrm{en}}$

3. Rabello LS. Promoção da Saúde: a construção social de um conceito em perspectiva comparada. Editora Fiocruz, $1^{\circ}$ Edição, 2010.

4. Ramos LR, Malta DC, Gomes GAO, Bracco MM, Florindo AA, Mielke GI et al. Prevalence of health promotion in primary health care units in Brazil. Rev Saúde Pública. 2014;48(5):837-44.

5. Gaff VA, Toassi RFC. Produção do cuidado em saúde com foco na clínica ampliada: um debate necessário na formação em Odontologia. Rev ABENO. 2017;17(4):6372.

6. Botazzo C. Sobre a Bucalidade: notas para a pesquisa e contribuição ao debate. Ciênc 
Saúde Coletiva. 2006;11(1):7-17.

7. Fonsêca GS, Botazzo C, Pires FS, Junqueira SR. Itinerário formativo em clínica ampliada: narrativas de uma cirurgiã-dentista. Rev ABENO. 2017;17(4):160-70.

8. Bastos BRMS, Clara AS, Fonsêca GS, Pires FS, Souza CR, Botazzo C. Formação em saúde bucal e Clínica Ampliada: por uma discussão dos currículos de graduação. Rev ABENO. 2017;17(4):73-86.

9. Merhy EE. Saúde: a cartografia do trabalho vivo. Hucitec, 2002.

10. Mafi A, Moretto C, Teixeira MFN, Saldanha OMFL, Rados ARV. A interdisciplinaridade e seus reflexo na formação do cirurgiãodentista. Rev ABENO. 2017;17(1): 62-71.

11. Moreira MCN. A construção da clínica ampliada na atenção básica. Cad Saúde Pública. 2007;23(7):1735-42.
12. Almeida JRS, Oliveira NC, Moura ERF, Saboia VPA, Mota MV, Pinho LGM. Oficinas de promoção de saúde com adolescentes: relato de experiência. Rev RENE. 2011;12:1052-8.

13. Fernandes MCP, Backes VMS. Educação em saúde: perspectivas de uma equipe da Estratégia Saúde da Família sob a óptica de Paulo Freire. Rev Bras Enferm. 2010;63(4):567-73.

14. Heidemann ITSB, Dalmolin IS, Rumor PCF, Cypriano CC, Costa MFBNA, Durand MK. Reflexões sobre o itinerário de pesquisa de Paulo Freire: contribuições para a saúde. Texto Contexto Enferm. 2017;26(4):2-8.

\section{Correspondência para:}

Janaína Rocha de Sousa Almeida e-maildrajanainarocha@ hotmail.com Rua Dr. Ratisbona, 171, Fátima 60.411-220 Fortaleza/CE 